\title{
Shape Tiling
}

\author{
Kevin Keating \& Jonathan L. King \\ keating@math.ufl.edu \& squash@math.ufl.edu \\ University of Florida, Gainesville FL 32611-2082, USA \\ Submitted: 28 July 1996 Accepted: 21 November 1996
}

\begin{abstract}
Given a list $1 \times 1,1 \times a, 1 \times b, \ldots, 1 \times c$ of rectangles, with $a, b, \ldots, c$ non-negative, when can $1 \times t$ be tiled by positive and negative copies of rectangles which are similar (uniform scaling) to those in the list? We prove that such a tiling exists iff $t$ is in the field $\mathbb{Q}(a, b, \ldots, c)$.
\end{abstract}

When can rectangle $1 \times t$ be packed by (finitely many) squares? DeHn 1903 gave the answer: If and only if $t$ is rational. For irrational $t$ he showed $1 \times t$ not packable by means of what we will call a "Dehn-functional". It is a map $\mathbf{D}$ from pairs of real numbers to $\mathbb{R}$ (or any abelian group) which satisfies:

$$
\begin{aligned}
& \mathbf{D}\left(\left[x+x^{\prime}\right] \times y\right)=\mathbf{D}(x \times y)+\mathbf{D}\left(x^{\prime} \times y\right) \\
& \mathbf{D}\left(x \times\left[y+y^{\prime}\right]\right)=\mathbf{D}(x \times y)+\mathbf{D}\left(x \times y^{\prime}\right)
\end{aligned}
$$

It is straightforward to check that for a packing of a rectangle $c \times d$ by finitely-many others, $\mathbf{D}(c \times d)$ must equal the sum of the functional applied to each rectangle in the packing. (The analogous statement applies to tiling. See the Definitions section, below, for a formal definition of packing and tiling.)

Two recent papers by Freiling \& Rinne 1994, and by LaczKovich \& Szekeres 1995, turn the question around: For which sidelengths, s, can the square be packed by rectangles similar to $1 \times s$ and $s \times 1$ ? Employing a Dehn-functional and a theorem of WALL 1945, they give this astonishing answer: Iff $s$ is algebraic over $\mathbb{Q}$, and all of its conjugates in the complex plane have positive real part. (We shall henceforth refer to such numbers $s$ as Wall numbers.)

Tilings. Every packing problem has an analogous problem using both positive and negative copies of the prototiles; we will call this operation "signed packing" or "tiling".

It turns out that Dehn's question has the same answer if tiling is allowed: $1 \times t$ can be tiled by squares iff it can be packed by squares. However, one sees readily that the [FR,LS] question has a larger answer if tiling is allowed, by considering the Golden Ratio

1991 Mathematics Subject Classification. 05B45. Secondary: 52C20 51M25 05B50 12 E99.

Key words and phrases. Tiling, packing, Wall number, Dehn's theorem, fields. 
$\lambda:=\frac{1+\sqrt{5}}{2}$. The conjugate of the Golden Ratio is $\frac{1-\sqrt{5}}{2}$, which is negative. Thus the [FR,LS] theorem guarantees that no square can be packed by rectangles similar to $1 \times \lambda$ and $\lambda \times 1$. Nonetheless, there is a tiling:

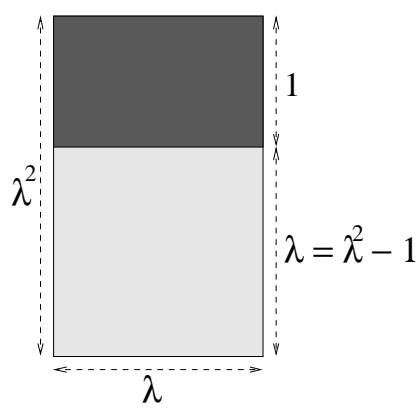

FIGURE 1 The dark rectangle, $\lambda \times 1$, is being subtracted from the top of the tall $\lambda \times \lambda^{2}$ rectangle. Since $\lambda^{2}$ equals $\lambda+1$, what remains after the subtraction is the $\lambda \times \lambda$ square.

The goal of our article is to establish a general tiling theorem for rectangles. A special case of the Tiling Theorem, below, is:

Rectangles with shapes $\{1 \times s, s \times 1\}$ can tile a square $\operatorname{IFF} s \in \mathbb{Q}\left(s^{2}\right)$.

Definitions. As usual, let $\mathbb{Q}(x)$ denote the field of rational functions of $x$, with coefficients in $\mathbb{Q}$. For $\zeta$ a complex number, $\mathbb{Q}(\zeta)$ is the smallest subfield of $\mathbb{C}$ containing $\zeta$. Given a (finite or infinite) subset $S \subset \mathbb{C}$, let $\mathbb{Q}(S)$ be the smallest subfield of $\mathbb{C}$ which includes $S$.

Identify a rectangle $a \times b$ with a product of half-open intervals, the subset $[0, a) \times[0, b)$ of the plane. A translate, $T$, of $a \times b$ is a set of the form

$$
\left[t_{1}, t_{1}+a\right) \times\left[t_{2}, t_{2}+b\right)
$$

where $t_{1}, t_{2} \in \mathbb{R}$. Say that a collection $\mathbb{P}$ of rectangles packs $c \times d$ if we can find a (finite) collection, TRANS, of translates of copies of rectangles in $\mathbb{P}$ such that we have equality

$$
\mathbf{1}_{c \times d}=\sum_{T \in \mathrm{TRANS}} \mathbf{1}_{T}
$$

between indicator functions. (Indicator function $\mathbf{1}_{T}$ is 1 for each point $(x, y)$ in $T$ and is 0 for all other points in the plane.)

Say that collection $\mathbb{P}$ tiles (or "signed-packs") rectangle $B=b_{1} \times b_{2}$ if: A finite collection TRANS and coefficients $\alpha_{T} \in\{1,-1\}$ can be found so that

$$
\mathbf{1}_{B}=\sum_{T \in \mathrm{TRANS}} \alpha_{T} \mathbf{1}_{T}
$$

(All of these definitions make sense in $D$-dimensional Euclidean space. For integer-sided $D$-dimensional polyominoes and bricks, this type of tiling question was studied by BARNES [B1,B2] and KING [Kin]. In particular, given a finite proto-set $\mathbb{P}$ of $D$-dimensional bricks there is an algorithm -which runs, as a function of the number of bits needed to describe a brick $B=b_{1} \times b_{2} \times \cdots \times b_{D}$, in linear time- to determine whether $B$ is tilable by $\mathbb{P}$. There is also a computable number $\mathcal{M}=\mathcal{M}(\mathbb{P})$ so that if each sidelength $b_{i} \geq \mathcal{M}$, then $B$ is $\mathbb{P}$-packable iff it is $\mathbb{P}$-tilable.) 
Lastly, a tiling $\mathbf{1}_{c \times d}=\sum_{T \in \text { TRANS }} \alpha_{T} \mathbf{1}_{T}$ is "horizontally splittable" if we can write $c=c^{(1)}+c^{(2)}$ and TRANS $=\mathcal{C}^{(1)} \sqcup \mathcal{C}^{(2)}$, a disjoint union of non-empty sets, so that:

$$
\mathbf{1}_{c^{(i)} \times d}=\sum_{T \in \mathcal{C}^{(i)}} \alpha_{T} \mathbf{1}_{T},
$$

for $i=1,2$. Define "vertically splittable" analogously.

Tiling (2) is completely-splittable if, either: TRANS is a singleton or -recursivelythe tiling can be split, either horizontally or vertically, into two tilings each of which is completely-splittable.

Shapes. Uniformly scaling rectangle $a \times b$ by scale-factor $u$ (a positive number) yields rectangle $a u \times b u$. Let the shape $a \times b$ represent the set of all uniform-scalings of the rectangle. Consequently, say that $\mathbb{P}$ shape-packs $c \times d$ if the union

$$
\bigcup_{a \times b \in \mathbb{P}}\{a u \times b u \mid u>0\} \quad \text { packs } \quad c \times d .
$$

Define "P shape-tiles $c \times d "$ analogously.

\section{$\S 2$ Some Results}

We start with a normalization. For each positive number $v$, a collection $\{1 \times s\}_{s \in S}$ shape-tiles $1 \times t$ iff $\{1 \times v s\}_{s \in S}$ shape-tiles $1 \times v t$. We can choose $v$ so that some product $v s$ is 1 . Consequently, we can assume, gratis, that $S$ contains 1 .

Tiling Theorem, 3. Suppose $1 \in S$, where $S$ is a (finite or infinite) set of positive reals. Then rectangles $\mathbb{P}:=\{1 \times s \mid s \in S\}$ shape-tile $1 \times t \operatorname{IFF} t$ is in $\mathbb{Q}(S)$, and $t \geq 0$.

Moreover, when $t \in \mathbb{Q}(S)$, there is a tiling which is completely-splittable and uses only scale-factors in the field $\mathbb{Q}(S)$.

Proof. For a tilable $1 \times t$, it will be temporarily convenient to say that $1 \times(-t)$ is tilable also. Definition (2) extends consistently to rectangles with negative sidelengths, if we identify $\mathbf{1}_{a \times(-b)}$ and $\mathbf{1}_{(-a) \times b}$ with $-\mathbf{1}_{a \times b}$. Thus we can freely remove the " $t \geq 0$ " in the statement of the theorem.

We will make use of the field $K:=\mathbb{Q}(S)$.

Establishing $(\Rightarrow)$. If $t \notin K$ then there exists ${ }^{\dagger}$ a $K$-linear functional $f: \mathbb{R} \rightarrow \mathbb{R}$ such that $f(t)=0$ and $f(1)=1$. Thus

$$
\mathbf{D}(x \times y):=x \cdot f(y)
$$

is a Dehn-functional. For any $s \in S$ and real $u$,

$$
\mathbf{D}(u \times s u)=u \cdot f(s u)=u \cdot s \cdot f(u)=\mathbf{D}(s u \times u) .
$$

\footnotetext{
${ }^{\dagger}$ We can define the linear functional by picking a $K$-basis for $\mathbb{R}$. Or, we can avoid the Axiom of Choice, as follows. Let $V$ be the $K$-vector-subspace of $\mathbb{R}$ spanned by the sidelengths of all the rectangles in the purported tiling. Extend the collection $\{t, 1\}$ to a $K$-basis for $V$, then define $f$ on this basis to get the desired $K$-linear-functional $f: V \rightarrow \mathbb{R}$.
} 
Thus the Dehn-functional $\mathbf{D}(y \times x)-\mathbf{D}(x \times y)$ is zero on every shape in the proto-set $\mathbb{P}$. Hence this Dehn-functional must be zero on each tilable rectangle. On the other hand, its value on $1 \times t$ is the difference $t \cdot 1-1 \cdot 0$, which is not zero.

Establishing $(\Leftarrow)$. Let $\mathcal{G}$, the "good set", be the collection of numbers $t$ such that $1 \times t$ is shape-tilable by the proto-set. Consider good numbers $p$ and $q$. Then $1 \times(-p)$ is tilable and, by stacking $1 \times p$ on top of $1 \times q$, also $1 \times(p+q)$ is tilable. Thus

The good set is preserved under negation and addition.

What happens when we place $1 \times p$ and $1 \times q$ side-by-side? Scaling each appropriately gives rectangles $q \times q p$ and $p \times p q$. These tile $(p+q) \times p q$. So if $p+q \neq 0$, we conclude that $\frac{p q}{p+q}$ is good. Thus

\section{The good set is preserved under "twisting"}

where, for $p \neq-q$, we define the twist of $p$ with $q$ to be

$$
p \bowtie q:=\frac{p q}{p+q} .
$$

Notice that the operation of twisting rectangles $1 \times p$ and $1 \times q$ scales them by scalefactors $\frac{q}{p+q}$ and $\frac{p}{p+q}$, both of which are in $K$.

Lastly, since the operation of twisting (resp. addition) corresponds to building a tiling which splits horizontally (resp. vertically), the following Field Lemma will complete the proof of the theorem.

Field Lemma, 4. Suppose $1 \in \mathcal{G}$, where $\mathcal{G}$ is a subset of $\mathbb{C}$ which is closed under negation, addition and twisting. Then $\mathcal{G}$ is a subfield of $\mathbb{C}$.

Proof. Suppose $p$ is "good", that is, in $\mathcal{G}$. Then $p n$ and $p / n$ are good, for positive integers $n$; this follows by induction and using that goodness is preserved under addition and twist. In the following, $p$ and $q$ are assumed to be good.

Reciprocals are good: For $p \neq 0$, note that $(p-1) \bowtie 1=\frac{p-1}{p}$ is good. Thus $\frac{1}{p}$, which equals $1-\frac{p-1}{p}$, is good.

Squares are good: $\quad$ Since $(1 \pm p)$ is good, $(1-p) \bowtie(1+p)$ is good. Multiplying by -2 yields that $p^{2}-1$ is good, hence $p^{2}$.

Products are good: $\quad$ Since $(p+q)^{2}-(p-q)^{2}$ is good, so is $4 p q$ and thus $p q$.

Addendum. Note that the lemma continues to hold with $\mathbb{C}$ replaced by any field whose characteristic is not two, i.e, $1+1 \neq 0$.

Question. By using a Dehn-functional, it is straightforward to see that if the tiling in Theorem 3 is actually a packing, then all the scale-factors must be in $\mathbb{Q}(S)$.

Does this same conclusion hold for all minimum-cardinality tilings? (I.e, those which minimize the cardinality of TRANS, the set of translates). 
Closing remark. The [FR,LS] theorem suggests studying the following transitive relation $\Rightarrow$ on the positive reals: $s \Rightarrow t$ if $\{1 \times s, s \times 1\}$ shape-packs $1 \times t$. Restating their result: $s \Rightarrow 1$ iff $s$ is a Wall number. Consequently, these numbers are hereditary; if $s \Rightarrow t$, with $t$ a Wall number, then $s$ is too.

We currently have no understanding of the arrow relationship. Certainly if the minimal polynomial of $s$ is unrelated to that of $t$, then there is no reason to expect $s \Rightarrow t$. Our theorem can, of course, give no positive result. It does, however, give the negative result that even if $s$ and $t$ have the same minimal polynomial, neither need arrow the other-simply because neither tiles the other.

In the normalization of the Tiling Theorem, a collection $\{1 \times s, s \times 1\}$ shape-tiles $1 \times t$ exactly when $s t \in \mathbb{Q}\left(s^{2}\right)$. Now suppose lengths $s$ and $t$ have a common minimal polynomial $f(x) \in \mathbb{Z}[x]$ which is cubic with three positive roots. Certainly st $\notin \mathbb{Q}\left(s^{2}\right)$ occurs if $\mathbb{Q}(s)$ fails to contain all three roots. And this will be the case if the discriminant of $f$ is not a perfect square. (See definition and corollary of [Jac, p. 258].) Indeed, we only need find such an $f$ with 3 real roots since, for a sufficiently large integer $T$, the translated polynomial $x \mapsto f(x-T)$ will have all roots positive.

An example is provided by $f(x):=x^{3}-6 x+2$, which has 3 real roots and, by the Eisenstein Criterion [H, Thm.3.10.2], is irreducible. The discriminant of $f$ equals $-4 \cdot(-6)^{3}-27 \cdot 2^{2}=6^{2} \cdot 3 \cdot 7$, which is not a perfect square.

\section{REFERENCES}

[B1] F.W. Barnes, Algebraic theory of brick packing, I, Discrete Math. 42 (1982), 7-26.

[B2] F.W. Barnes, Algebraic theory of brick packing, II, Discrete Math. 42 (1982), 129-144.

[Deh] M. Dehn, Über die Zerlegung von Rechtecken in Rechtecke, Math. Ann. 57 (1903), 314-332.

[FR] C. Freiling \& D. Rinne, Tiling a Square with Similar Rectangles, Math. Research Letters 1 (1994), 547-558.

[H] I.N. Herstein, Topics in Algebra, Wiley \& Sons, 1975.

[Jac] Nathan Jacobson, Basic Algebra I, 2nd ed., W.H. Freeman, 1974.

[Kin] J.L. King, Brick Tiling and Monotone Boolean Functions, Preprint available at webpage http://www. math.ufl.edu/ squash/

[LS] M. Laczkovich \& G.Szekeres, Tilings of the Square with Similar Rectangles, Discrete Comput. Geom. 13 (1995), 569-572. 